\title{
LA GASTRONOMÍA BIEN TEMPLADA. LA COCINA QUECHUA
}

Julio Calvo Pérez*

\section{EL PAR WAYK'UY / YANUY}

Un acercamiento al léxico sobre cocina en lengua quechua nos podría proporcionar algunas claves sobre su importancia en tiempos del imperio inca y sobre la que tiene, en el momento presente, la cocina patrimonial del Perú'. No hace mucho un hablante de quechua nos decía convencido: "Piru llaqta wayk'uyninqa sumaqnin" ("la cocina de Perú es realmente exquisita").
Llama en primer lugar la atención la existencia de dos lexemas en quechua para indicar la acción general de cocinar ${ }^{2}$, los cuales, a la vista de la documentación obtenida (Calvo, en prep.) distan de ser sinónimos absolutos:

1) Wayk'uy es el término general para 'cocinar', como se desprende de la siguiente entrada y subentradas derivadas de ella, acomodadas a las pretensiones del momento:

\begin{tabular}{|c|c|}
\hline \multirow{2}{*}{ 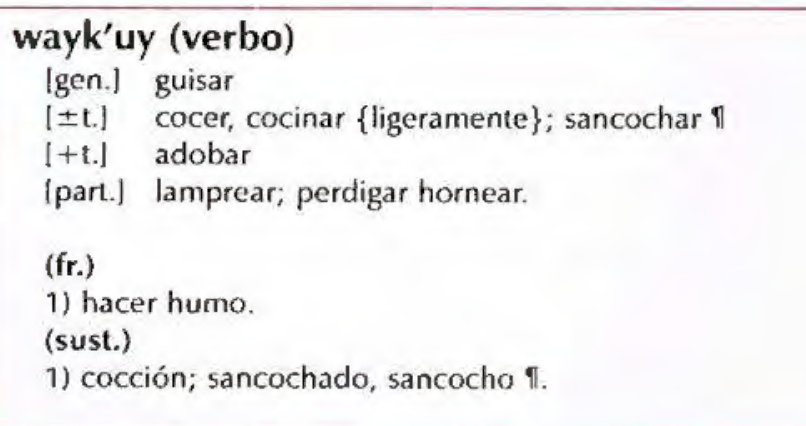 } & $\begin{array}{l}\text { wayk'uq } \\
\text { [gen.] cocinero } \\
\text { [ } \pm \text { neg.] ranchero } \\
\text { [+neg.] marmitón. }\end{array}$ \\
\hline & $\begin{array}{l}\text { wayk'u } \\
\text { (sust.) } \\
\text { 1) [ } \pm \text { cant.] (chayay), cochura, cocción } \\
\text { 2) cocido, sancochado. } \\
\text { (adj.) } \\
\text { culinario. }\end{array}$ \\
\hline $\begin{array}{l}\text { wayk'uynin } \\
\text { 1)[-mat.]cocina. }\end{array}$ & $\begin{array}{l}\text { wayk'ukunapaq } \\
\text { 1) (mankakuna), ollería. }\end{array}$ \\
\hline $\begin{array}{l}\text { wayk'una } \\
\text { [loc.] fogón; placa } \\
\text { I+cant.](wayk'una wasi), cocina, lugar \{para cocinar\} } \\
\text { linstr.] combustible. }\end{array}$ & $\begin{array}{l}\text { wayk'uchiy } \\
\text { 1) disponer, hacer }\{\text { la comida }\} \text {. }\end{array}$ \\
\hline $\begin{array}{l}\text { wayk'una ukhu } \\
\text { 1) sauseria. }\end{array}$ & $\begin{array}{l}\text { wayk'uchina } \\
\text { 1) (t'uqtina), atizador, badil. }\end{array}$ \\
\hline $\begin{array}{l}\text { wayk'unapaq qillqasqa } \\
\text { 1) (risita), receta \{de cocina\}. }\end{array}$ & $\begin{array}{l}\text { wayk'upakuq } \\
\text { 1) sollastre. }\end{array}$ \\
\hline
\end{tabular}

- Linguista, profesor de la Universidad de Valencia (España)

1 Véase, al respecto, el estudio sobre las fuentes léxicas clásicas realizado por Antúnez de Mayolo (1981). Para el conocimiento de los rasgos mestizos debidos a la aportación espanoola, cfr:: Olivas Weston (1996).

2 Casi todos los diccionarios recogen esta duplicidad, desde los más antiguos (Santo Tomás 1560, González Holguín 1608) a los actuales (Lira 1944), pasando por los alema nes del siglo XIX (Tschudi 1853, Middendorf 1892). Estos autores, por la amplitud de las entradas, dan mayor juego a wayk'uy, dejando como más especializado a yanuy. 
Se observará, antes de nada, que no es preciso recurrir a ningún préstamo del español o del inglés para hacerse cargo de los términos principales que implican la noble actividad de dar de comer a la familia, a los clientes o, esporádicamente, a los invitados. No obstante, el préstamo risita "receta" nos pone en guardia sobre la sistematización a que hoy día está sometida la acción culinaria: la necesidad de inventar, el deseo de perpetuar los hallazgos está dando lugar ya, en algunas partes del mundo, a que las recetas tengan nombre propio: el del cocinero que las generó. La palabra compleja quechua wayk'unapaq qillqasqa alude, por su parte, al "escrito con instrucciones para cocinar", lo que en tiempos pasados sería meramente el yuyariy, el "recuerdo" transmitido de boca en boca y de generación en generación de cómo elaborar los distintos platos, en qué fechas hacerlos y con qué ingredientes confeccionarlos, dependiendo de las disponibilidades del lugar y del momento. Destaca el hecho de que este término, al ser tan general, se refiere a cualquier actividad culinaria, cualquiera sea su grado de especialidad: cocinero es no marcado, pero ranchero está algo degradado y marmitón soporta una carga mayormente negativa. La frase verbal "hacer humo" es incluso peyorativa.

2) El segundo término yanuy es meliorativo, como apreciamos en seguida:

\begin{tabular}{|c|c|}
\hline $\begin{array}{l}\text { YANUY (verbo) } \\
\text { 1) [alim.] } \\
\text { 2) [+t.] cocer, } \\
\text { 3) [part.] }\end{array}$ & $\begin{array}{l}\text { cocinar }\{\text { con arte }\}, \text { restaurar } \\
\text { cocinar }\{\text { perfectamente }\} \\
\text { (qaray), servir }\{\text { la comida }\} \text {. }\end{array}$ \\
\hline $\begin{array}{l}\text { YANUQ } \\
\text { 1) maestresala. }\end{array}$ & \\
\hline $\begin{array}{r}\text { YANU (yanun) } \\
\text { 1) "cult.», come } \\
\text { qque se }\end{array}$ & $\begin{array}{l}\text { ble } \\
\text { one a la mesa en una porción }\end{array}$ \\
\hline
\end{tabular}

\begin{tabular}{l} 
YANUKUY \\
hora \{de cocción\}, rato. \\
\hline $\begin{array}{l}\text { YANUKUQ } \\
\text { 1) (wayk'uq), cocinero; «mod.», restaurador. }\end{array}$ \\
\hline
\end{tabular}

Las equivalencias no albergan ningún género de dudas: el arte se ha instalado en la cocina con esta precisión léxica. El sinónimo de wayk'uy sería innecesario, al ser este más general, pero al surgir como matiz sobre él permite en quechua cuzqueño las lecturas ponderativas hechas. En su origen yanuy es dejar bien cocinado, con cocción incluso pasada, pero por eso mismo implica tiempo: el que se dedica a hacer las cosas con cuidado, cuya carencia nos llevaría a obrar para salir del paso, a preparar algo sobre la marcha para alimentarse, al extremo del wayk'uy, que puede significar "preparar ligeramente". Así pues, una frase nominal como Yanusqa papa "papa cocinada" no supone un mero sancochado, cocido a medias o simplemente cocido, sino una preparación especial por su sabor y su acabado; como la actividad artística, efímera pero genial al mismo tiempo, de hacer arte con las sustancias menos rígidas; de realizar esculturas casi horizontales con los productos más perecederos; de tallar a golpe de cuchillo y tenedor frutas y hortalizas, carnes y pescados, de bordar con la manga pastelera en la mano la enjundiosa repostería... A diferencia de yanuy, wayk'uy tiene una disposición inmediata a entenderse como "cocer" y podría asumirse, desde el principio, como palabra más propia para preparar potajes o cocidos ${ }^{3}$.

La palabra yanuy, con implicaciones aparentemente modernas, es más bien el término panquechua para referirse a la actividad de la coci$n a^{4} y$, aunque en el pasado inca no existía el oficio $^{5}$ de cocinero, la palabra tenía connotaciones positivas ${ }^{6}$, las cuales se desarrollan sobre todo en el dialecto cuzqueño, una vez que se produce la duplicidad sinonímica con wayk'uy?

3 La palabra wayk'uy existe también en aimara (Bertonio 1612) con idéntico significado: "cozida, tiempo en que cueze algo: phaa, vel huayku", aunque el témino se emplea, sobre todo, para 'teñir'.

4 Por ejemplo, el Vocabulario poliglota incaico (VPI 1905) acepta yanuy y yanukuy no solo para el ayacuchano y el cuzqueño, sino también para las lenguas quechuas de Áncash y Junín. Lo mismo puede decirse para Huánuco (Weber et al. 1998), Tarma (Adelaar 1977) y Cajamarca (Quesada 1976), etc.

5 Es lo que asegura con frecuencia Olivas (1996). No obstante, Domingo de Santo Tomás recoge yanucamayoc "cozinero" y no debemos olvidar que la presencia de kamayuq (como en khipu kamayuq "quipucamayo" o "experto en quipus") no implica mero oficio, sino experiencia o acendramiento en la actividad a que se refiere, cuando no rango superior en la actividad ("oficial") y, más aún, creatividad (Calvo, s.v, kamay). Parece lógico aceptar entonces que la cocina era un arte en la filosofía en lengua quechua (Antúnez de Mayolo 1981: 136).

6 En el diccionario de Lira, por ejemplo, leemos: "YANU - YANUN: Postre o comestible que se pone a la mesa para que el comensal se sirva a discreción* $/$-YANUKK Iyanuq]: Maestre de cocina. Maestresala, oficial que presenta y reparte manjares en las mesas de rango". El término correlativo wayk'uq representaría apenas a la persona activa en la cocina: "cocinero, cocinera, persona que entiende de culinaria y se ocupa de cocinar", como señala Lira (1944).

7 Duplicidad sinonimica que, no hay que olvidar, se venía dando desde el pasado más remoto. En el primer vocabulario español-quechua de 1560 , Domingo de Santo To más recoge: "Cozer: yanucuni. gui, o guaycuni. gui". Yaranga (2003) resuelve también el par -en ayacuchano- en favor de yanuy, puesto que en wayk'uy añade: "este verbo puede significar cocinar ligeramente* (la cursiva es nuestra). 


\section{EL TIEMPO DE COCCIÓN O YUPAY}

Obsérvese, además, que la duración estricta de la cocción da lugar a una unidad de medida temporal, el yanukuy, equivalente a una hora de reloj en circunstancias estándares. De hecho la importancia reside, para muchos, en que el mundo andino proporcionaba "la base de una cultura intimamente ligada al alimento" (www.interactive.net.ec)

"Tanto así que una de las unidades de medida de tiempo que tuvieron nuestras culturas era el lapso en que se demoraba en cocer un tipo de papa" (ibid.).

La preparación de la piedra de la pachamanca dura aproximadamente también ese tiempo (no menos de una hora): "Hacemos un hoyo regular en la tierra, generalmente de setenta centímetros de diámetro y cuarenta centímetros de profundidad, para una pachamanca de diez a quince personas. Colocamos unas cuantas piedras limpias en el fondo como una primera capa. Luego, calentamos las piedras en una fogata a alta temperatura durante por lo menos una hora" (www.saboresdelpderu.com).

$Y$ es probable que muchas otras actividades supusieran partes divisas del yanukuy o múltiplos de ella, ya que sería conveniente poder servir al punto los alimentos (qaray) en sus porciones y ritmos oportunos. Lo que para los occidentales es el tiempo de un huevo pasado por agua u otras medidas fijas, para los andinos era sin duda el yanukuy. De ese modo, la simple medida a ojo o por tanteo (tupuy) da lugar a una medida de precisión más ajustada a la cuenta atrás, para que las viandas estén a tiempo (yupay).

\section{Los conceptos involucrados respecto del par wayk'uy / yanuy}

En tiempos antiguos, en referencia a las actividades artísticas de la confección -frente a las artesanas-, estaba también implicado el concepto de yanuy, que venía a encerrar los actuales de "diseño"8. Lo mismo podría decirse, directamente, de la actividad culinaria, la "restauración" en que se centra este trabajo. A tenor de lo observado y dado el carácter ennoblecedor de la palabra yanuy "cocinar", convendría detenernos a observar a qué tipo de conocimiento correspondería la actividad en estudio.

Haciendo una valoración epistemológica, hay que distinguir en quechua una serie de verbos que corresponden a otras tantas actividades del ingenio, la mente y la mano de los hombres andinos en una escala que va de lo más concreto a lo más abstracto, como ya sucedía entre los griegos. Entre estos, el arte, la ciencia y la técnica eran los tres pilares de la epistemología de la creación, los cuales han pasado al mundo occidental con intereses cambiantes, como mostró Trier $(1931)^{9}$. En quechua las cosas son de un modo ligeramente diferente, aunque podemos establecer alguna correspondencia. Independientemente de los cambios habidos, los conceptos que se manejan en el mundo andino son los siguientes:

8 El yanu p'acha kamayuq "oficial \{de ropa basta\}" se distinguía del yanu pawqar kamayuq "oficial \{de ropa fina\}" (el modisto o diseñador de ahora) o también "oficial \{de plumeriał", en que se especulaba no tanto entre una actividad pobre y desmantada frente a otra sublime y artistica, sino en que se evaluaba ba confección de telar, como actividad noble, que servía para vestir al pueblo, desde los yanakuna a los qhapaghuna ("pobres" y "ricos"), en oposición a la verdadera ciencia de la confección, en vicuña o guanaco, con que se vestía el inka, la quya ("reina") y las nust'akuna ("princesas") reales. Igualmente se implicaba en ello la ciencia del omato, ya haciendo pillu ("guirnalda") bien entretejido mediante el arte del ch'antay ("adorno foral"), ya recurriendo a las plumas multicolores de las aves autóctonas: del flamenco rosado o pariwana, del pilco empleado en los sacrificios (pillqu o pillhu) o del mitico corequenque (qiriqinqi: "buitre \{pequeño, de cuello pelado\}" que se cazaba también especialmente para las mejores ocasiones. En todo caso, el tejido era un arte en el que el qunpiy ("bordado, recamado") y el pallay ("tejido de filigrana"), los célebres tukapu ("labores, entredoses del jubón", que dieron nombre a un diosl, propiciaron la adniración temprana de los invasores españoles. Obsénese bien el complejo léxico y el elemento que distingula las labores: en el primer caso p'acha es "vestido"; en el segundo pawqar es "color" $y$ "matiz"; en ambos, yanu muestra la nobleza de la actividad textil y kamayuq el "oficio", la "pericia" en la realización de la actividad emprendida.

9 Para el autor alemán, hacia 1200 la palabra del mundo intelectual wisheit "sabiduria" recubria el significado de kunst "arte" (saberes caballerescos sistemáticos y amónicos) y list "astucia" (saberes asistemáticos que se adquirian por la experiencia, propios del picaro). Un siglo después, los conceptos de "sabiduria" $y$ "arte" competían en pie de igualdad con wizzen "conocimiento". A esto podemos anadir que la "astucia" se seguiría empleando como fondo de la actividad consuetudinaria de caracter social, de modo que abandonar esta sería como abandonar la lucha por la subsistencia en tiempos dificiles. 


\section{EL KAMAY}

En primer lugar existía el kamay "creación", a partir de la cual se desarrollaba un conocimiento de "experto" a cargo del kamayuq, cuya traducción, a tenor del despliegue polisémico, podría ser la que sigue:

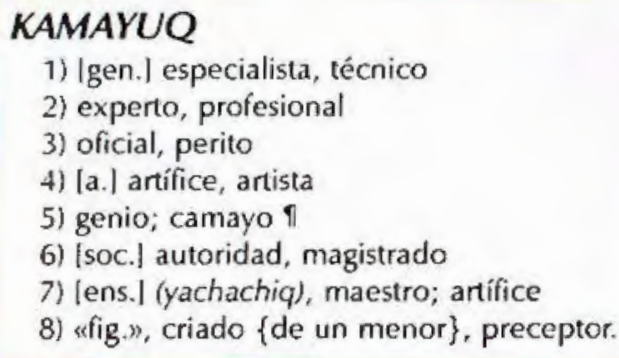

1) Igen.I especialista, técnico

2) experto, profesional

3) oficial, perito

4) [a.] artífice, artista

5) genio; camayo १

6) [soc.| autoridad, magistrado

7) (ens.l (yachachiq), maestro; artífice

8) «fig.", criado \{de un menor\}, preceptor.

De todas esas acepciones, la de "autoridad" es seguramente de las más antiguas. Refleja la idea de que, socialmente, la autoridad viene de la capacidad para tenerla, demostrada por la acción. Todas evidencian, de cualquier manera, como ha mostrado Taylor (1974), la vitalidad, la fuerza noble del animador, descubiertas en kamay (1) -más abajo. Y también la del creador, pese a lo que dice el autor anterior: el artista, cuyo genio permite idear cosas con originalidad y transmitirlas a los demás (yachachiy), ya que no crear en el sentido divino de sacar de la nada ${ }^{10}$. La palabra camayo T es un préstamo del quechua a la cultura universal, una palabra valiosa en cuanto que abarca múltiples posibilidades experienciales: frente a la actividad especulativa del sabio filósofo y pensador (hamawt'a), que ha dado el préstamo correspondiente amauta I, aparece esta con sus implicaciones praxeológicas incluidas.

Por otra parte, las ideas encerradas en (3) son las que mejor se aproximan a la idea de creación por parte de los humanos, cuyos bosquejos mentales o físicos nos aproximan a los dioses, tanto que nosotros mismos hemos inventado a Dios, al menos al dios de la cocina, a nuestro modesto entender. A nosotros no nos toca directamente (2) producir de la nada, pues dependemos de los productos que da la tierra, el cielo y el mar, pero modelamos o plasmamos los platos o damos el sabor justo tanto al aqha (la chicha indígena, que en el mundo antiguo era aswa) como al winu (el vino occidental). Pero nos toca también (4), ya que obligamos a la materia prima a organizarse en sí misma (5), ya que planteamos el cruce de sabores que llega a nuestro paladar, apoyados en (6), la penetración que nos posibilita lograr nuestros objetivos mediante la cabal combinación de ingredientes (7). Eso es la cocina.

Por otro lado (2) es más bien una interpretación occidental de kamay, que no sirve bien a nuestros propósitos, pero que no invalida que el cocinero de altos vuelos, el yanukuq, pueda llegar a cotas sublimes en el manejo de unos productos paradójicamente tan perecederos. Afortunadamente, no se vive del aire ni de la nada y el pasto espiritual no está reñido con el material. Justamente hemos pasado por los siete grandes conceptos de kamay que son, analíticamente, los que figuran a continuación (Calvo en prep.):

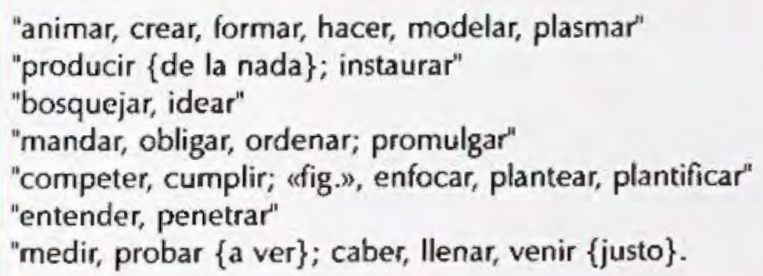

Para González Holguín, kamay representaba, en síntesis, el oficio, el saber y el talento, hechos concreción en la tarea, como explica el autor en las entradas correspondientes:

Camay ñiyquicama: Según tu oficio, o tu sauer, o talento.

Camay, o suyuy, la tarea en el trauajo.

Esas tres palabras revierten en quechua a riqsiy, a yachay y a yuyay respectivamente; es decir a la práctica, a la técnica y a la sabiduría de la cocina. Ahora veremos hasta qué punto el misionero jesuita estaba acertado en su apreciación. 


\section{EL YACHAY}

Si bien la cocina es creación o kamay, si bien puede llegar a improvisarse el acto culinario, algo que tantas veces hacemos por falta de tiempo, sumidos en el quehacer práctico de cada día, la cocina necesita de saberes cada vez más sistematizados en la perspectiva de eso que llamaríamos un saber "teórico-aplicado" o un conocimiento "práctico con ribetes de sistematización". Eso es lo que se llama el yachay, la ciencia del saber que se aboca a la disciplina aplicada emanada del kamay. Se trata de dos actividades que se mueven entre el conocimiento muy práctico del hacer consuetudinario (riqsiy "conocer"), con el que se comienza el aprendizaje del fogón, la tullpa andina que permite la cocción refractaria, y la pura teoría, el saber especulativo de los andinos, su filosofía (yuyay "pensar").

Si examinamos la entrada del diccionario de Calvo (en prep.) para ciencia -más abajo-, entenderemos que el cocinar convive de modo equilibrado con esos cuatro pilares: desde el mero wayk'uy, que se polariza entre el riqsiy y el kamay, al más sublime yanuy, cuyos extremos oscilan entre el kamay y el yachay. Evidentemente, el placer espiritual no está reñido con el material, pero el pensamiento profundo, enmarcado en el quehacer especulativo del yuyay, no se aviene simultáneamente con el acto emotivo del convivium; estos son sucesivos: quien no come bien, mal ha de reflexionar bien a su tiempo y quien no tiene capacidad de magín difícilmente saboreará a placer el néctar y la ambrosía de los dioses.

Pero cada cosa a su tiempo. Por eso no nos gustan las puestas en escena de las comidas de negocios como tales ni las comidas de los políticos. Los banquetes están bien para buscar la debida empatía antes o después de los actos económicos o de gerencia serios, pero nunca durante el proceso especulativo en que estos tienen lugar. Los amantes, lo mismo: cuando comen juntos en un lugar romántico o en el rincón más aislado del comedor lo hacen para fortalecer su amor, de modo afectivo, no para discutir sus diferencias o el modo de afrontar su proyecto: eso produce a la larga malas digestiones. Así, a la luz de las velas que se consumen lentamente, brindan, saborean los manjares y todo funciona; en cambio, reflexiones más profundas y trascendentales llevan, durante la hora y media en que el uno al otro se miran a la cara, a la ruptura, a soplar o apagar las velas de un manotón sin demasiadas contemplaciones.

Pero volvamos a la ciencia.

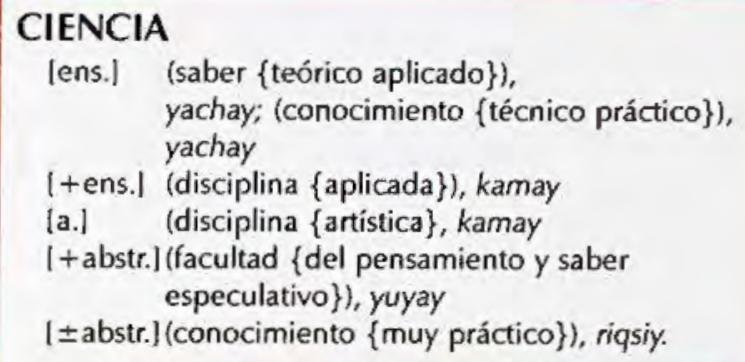

En efecto, la acción a mitad de camino entre el conocimiento teórico y el práctico que representa yachay "saber" implica el proceso diseñado en el kamayuq, cuya disciplina es la de aplicar esos conocimientos mediante la creatividad y el desarrollo cultural hereditario. Orlando los extremos de las ciencias se halla el yuyay "pensar, reflexionar" y el riqsiy "conocer por la experiencia": el primero nos lleva a los saberes sistemáticos de la antigüedad y más tarde a los experimentales, que permiten repetir el proceso alterando alguna variable y midiendo los resultados, mientras que el segundo lo hace remitiéndonos a la actividad de cada día, al ejercicio de ensayo y error por el que nuestras madres aprendieron a decantar sus exquisitos platos de guisadoras consuetudinarias. Puede que no haya cocinero profesional que alquitare así sus creaciones: la suya, la de ellas, es la fuerza de la tradición precisa en su variedad. Pero es también la repetición sin término, la extensión sin límites de un proceso que implica cada vez un poco más de lo mismo. Por eso tiene también la limitación de la falta de experimentalidad, de la incapacidad del avance que da el kamay, aunque kamayuq podrá ser también cualquier cocinero (o cocinera) dotado de la suficiente creatividad, aquel que se cree en su propia potestad para ejercer el yanuy, elevándose con la práctica de cada día al plano profesional.

Así se comprende por qué fallan muchos restaurantes, por qué un profesional, mitad artista $y$ mitad ejecutor diario, de ninguna manera se improvisa. 


\section{EL YUYAY}

El yuyay representa las distintas teorías sobre la cocina. Para los andinos precolombinos parte de la ciencia teórica se gastaba en experimentar los modos de conservación de los alimentos en los lugares donde escaseaban $y$, por supuesto, en la debida "docilización" de los frutos silvestres (sach'a) que debían ser cultivados sistemáticamente y mejorados en sus condiciones fitotécnicas. Así se obtuvo el tocosh, el chuño o se aprendió, no sabemos de quién, el proceso de fabricación de la chicha. Hay que hacer sin embargo un comentario: cuando se habla de comida bien equilibrada entre los habitantes prehispánicos en el Tawantinsuyo no se hace a veces más que propaganda utópica: todos los pueblos se esfuerzan por alimentarse y el andino no iba a ser una excepción, pero al lado de los muchos aciertos, señalados por diversos autores (Valcárcel 1949, Murra 1975, Espinoza 1987), a veces acríticos, están los estudios bien fundamentados que han hecho otros (Inca Garcilaso 1609, Malpica 1966, Zubritzki 1975) y que en conjunto obligan a pensar que los conocimientos iban siendo los apropiados, pero que faltaba aún mucho camino por recorrer: herramientas obsoletas, terreno en ocasiones inaccesible, clima riguroso, carencia de proteínas, etc.

Para el hombre medieval y renacentista, la teoría consistía en poder combinar adecuadamente el alimento frío con el cálido, como se hace en la pintura con los colores, con el fin posterior de buscar el equilibrio alimentario, de balancear los distintos alimentos según sus propiedades aparentes y mejorar o preservar, en definitiva, la salud. A la llegada de los españoles al Perú, estas ideas se transmitieron también al nuevo espacio culinario y se comenzó a clasificar los alimentos según su procedencia y cualidades (Ortega 1980, Olivas 1998).

Posteriormente el yuyay ha evolucionado a la dietética, a los alimentos altos y bajos en calorías, al consumo moderado de grasas, etc., lo que en el mundo entero culmina en lo que se viene llamando la dieta mediterránea, abundante en frutas $y$ verduras, en cereales y leguminosas, a favor del pan y el vino (evangélicos) en las comidas y del aceite de oliva en el proceso de cocción, una cocina antes más propicia al pescado que a la carne propiamente dicha. Esta es, sin duda, una gran aportación (Grande Covián 2000), pero hay también elementos que la disturban como el poco tiempo de que se dispone al mediodía para la comida principal, la aceleración o estrés con que se consume cualquier comida rápida, los productos químicos que adulteran los productos bajo la necesidad de su edulcoración y conservación, la maduración precoz en cámaras y tantos otros. En estas disposiciones sí que parece que nos superaban a veces los antiguos.

\section{LA COCINA ES UN ARTE}

De lo dicho hasta aquí se desprende que el yanuy es un arte y el yanukuq o restaurador un artífice, frente al wayk'uq "cocinador", que se encuentra un punto más distante en la escala que va de lo teórico a lo práctico. Véase en la siguiente entrada cómo armoniza la actividad artística con las reglas diseñadas para el arte de la buena cocina:

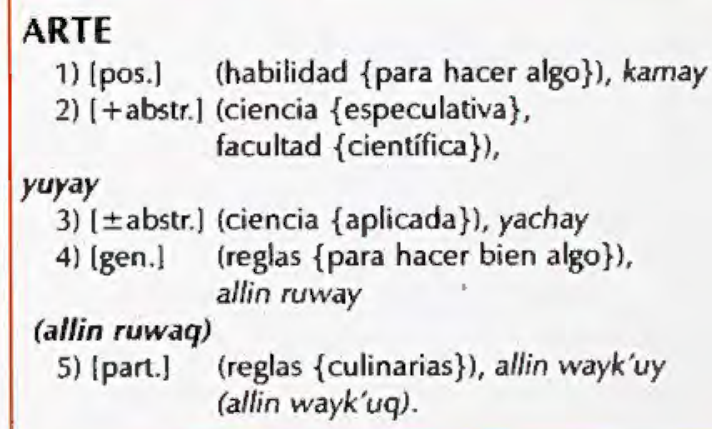

1) [pos.] (habilidad \{para hacer algo\}), kamay

2) [+abstr.] (ciencia \{especulativa\}, facultad \{científica\}),

En ella percibimos, en efecto, que el allin ruway ("el bien hacer") tiene que ver en general con la artesanía; de ahí que allin wayk'uy, potenciado con allin "bien", defina al artesano, el ser humano que maneja bien sus manos (o allin makiyuq runa) a la hora de preparar platos que han de consumir los demás o bien él mismo y los suyos. No obstante, la idea de artesano podría traernos reminiscencias de actividad de segunda categoría, actividad que hoy en los países civilizados realizan solo los emigrantes $y$ otros desheredados de la fortuna. Esta es, sin embargo, la base: el trabajo de cada día hecho con las manos del hombre y de la mujer, al 
margen de la comida horrible que hacen las máquinas ${ }^{11}$ y que ya sabemos encontrarla también en restaurantes de cadenas muy conocidas. De hecho, la cocina traída por los negros africanos (como el taku taku "mezclado" de arroz y frejoles, pese a su nombre quechua) o la misma que realizaban las indias al servicio de los españoles en la colonia (como el chunchuli bien tostado), son el puente que nos falta para aprovechar unas raíces que nos permitan crecer junto a otras influencias en el mundo de los fogones. Luego, del wayk'uy del artesano pasaremos al yanuy, como ya se dijo, y entonces el cocinero se tornará en artífice. Las entradas no dejan lugar a dudas:

\section{ARTESANO}

1) [hum.] (persona \{que elabora productos con un arte mecánico\}), allin makiyuq runa.

\section{ARTESANÍA}

1) labstr.] (arte \{de hacer algo a mano\}), allin ruway (allin makinwan nuway).

\section{ARTÍFICE}

\author{
1) (artista), kamayuq \\ 2) [ \pm cant.] (maestro \{que realiza una obra mecánica\}), \\ kamayuq \\ 3) «fig." (autor), kamachiq.
}

Nadie podría ser rotundamente lo segundo (un artífice) $\sin$ haber sido lo primero (un artesano), pero a lo segundo, al kamayuq, no todos están destinados. La noble artesanía de alimentar a la familia es la raíz del nobilísimo arte del "buen yantar": quien no haya pasado por ese tamiz tal vez no deba ser clasificado nunca en el ramo de lo segundo; y decimos esto por más que le pese a algunos. Pero al mismo tiempo, como dijimos arriba, eso no garantiza el salto cualitativo: podemos estar repitiendo siempre lo mismo sin salir un ápice de la rutina. $Y$ así no se llega a ser un genuino artífice. Por otro lado, el mero esnob, el cantamañanas de turno de la cocina, no es sino un impostor al que se descubre pronto.

Del mismo modo que un pintor que maneje con habilidad los pinceles necesita haber proyectado su cuadro y haber dibujado previamente las figuras o haberlas degradado a la percepción abstracta, así el cocinero debe haber planificado su plato, dotándolo de unas líneas maestras de sabor $y$ de olor, de color $y$ textura. $Y$ para esto necesita haber encallecido sus manos con las quemaduras del fuego, haber sentido paso a paso una cocción, haber visto combinar y combinado los sabores de toda la vida, que después de todo encierran un amplio saber cultural insustituible, el allin ruway (allin makinwan ruway). Así, de la continuidad del artesano en el wayk'uy se puede saltar, por arte de magia, a la discontinuidad del yanuy, una discontinuidad que no implica romper con la tradición, pero tampoco aceptarla corno si de un dogma más se tratara.

\section{LA COCINA ES UNA TÉCNICA}

En segundo lugar, la cocina es una técnica, techné, lo mismo que lo era hacer diccionarios para los dominicos o los jesuitas, autores también de espléndidas artes de la gramática. Recordemos de paso que una cosa no está reñida con la otra.

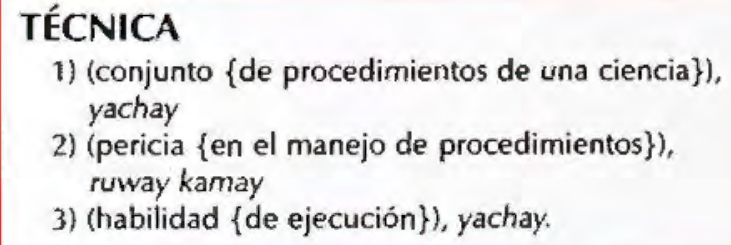

1) (conjunto \{de procedimientos de una ciencia\}), yachay

2) (pericia \{en el manejo de procedimientos\}), ruway kamay

3) (habilidad \{de ejecución\}), yachay.

\section{TÉCNICO}

1) (relativo a las aplicaciones de las ciencias\}), yachaq

2) (perito \{de la técnica\}), kamayuq

3) (conocedor (de la teoría\}), yachaq

La entrada que se acaba de cerrar implica dos bucles interesantes que se abren y cierran con el verbo yachay. Si vimos antes que la cocina es yachay "saber interiorizado", pronto comprenderemos que el cierre del círculo es lo que implica dedicarse a la cocina como uno de los más nobles saberes del mundo, ya que en su interior alberga la pericia del kamay como un "hacer" fundamental (nuway), un hacer que ennoblece al hombre hasta los límites de la creación (kamay) a partir de algo: de la materia prima ennoblecida. 
En definitiva, que la cocina es un arte, ya que la conjugación de los dos polos lleva a eso y eso es el yanuy. Como fondo explicativo se hallan las reglas del buen hacer, de la técnica, sin las cuales nadie puede llamarse, en esencia, cocinero. $Y$ ahí es donde entra, con toda propiedad, como puente entre ambos mundos, el kamay.

\section{LA CONCRECIÓN DEL ARTE CULINARIO ANDINO: EL RIQSIY}

Aunque un poco marginado, como el list "astucia" del análisis de Trier, el riqsiy "conocer" es el principio de todo artífice ${ }^{12}$ : la cocina inca debió enriquecerse conforme el imperio se expandía y eran conocidos nuevos modos o formas de preparación de los alimentos o se innovaba sobre el pasado ${ }^{13}$. Del alimento crudo al cocido se produjo una revolución, aunque el cebiche (palabra compuesta del español "cebo" más el sufijo causativo quechua -CHI: sibichiy "hacer cebo"), plato nacional peruano, consista en la degustación de pescado fresco, macerado con limón "redondo" y cebolla, sal y ají. Así se comenzaron a preparar alimentos cocidos bajo tierra, al contacto de piedras areniscas de fuerte poder refractario: la pachamanca (o bien pacha manka que significa "olla de tierra") es el gran plato elaborado de este modo, aquel en el que la convivencia familiar, el ágape de amistad, se hace imprescindible para que pueda ser degustado, pasando por la más humilde guatia (wathiya), esas hortalizas y tubérculos asados en contacto con piedras y con la tierra descolorida, endurecida por el calor ( $\left.t^{\prime} u q r a\right)^{14}$.

Al riqsiy pertenecen también los recetarios, aunque se ha de procurar tomarlos como referencia y no siempre como modelos dogmáticos a seguir. Tal vez una paella valenciana se deba a un ritual y a unos ingredientes bastante ajustados: el arroz "bomba", el pollo y el conejo, los tres tipos de judías: la judía verde (ferraura), la judía grande y seca (garrofón, similar al pallar peruano), la judía blanca tierna (tave(Ia), un poco de tomate y el imprescindible azafrán, hecho todo según los cánones con leña de naranjo. Luego, sobre la base que conforman los platos, las recetas cubren infinidad de variantes donde se mezcla la creatividad con la experimentalidad más libre (hemos leído de paellas hechas incluso con jamón y calabacín, cebolla, queso, etc. cayendo en la más absurda de las heterodoxias) ${ }^{15}$.

No vamos a entrar aquí en aspectos como la psicología de los pueblos ni zarandajas de este estilo: el turismo, los constantes viajes de negocios, las excursiones culturales evidencian que la cocina se va haciendo cada vez más mestiza, lo cual se suma a que cada vez con más frecuencia se instalan restaurantes de las más diversas nacionalidades en nuestros hábitats. En ocasiones los cocineros buscan la idea pura; en otros casos se da el oportunismo que resulta de trivializar para adaptarse a los gustos del país y a los de los circunstanciales comensales que acuden a su llamada; y esto es malo. Pero lo otro, la búsqueda de nuevos sabores y formas, nos hace cada vez de paladar más exigente, compartiendo el riqsiy, el "conocer" con los mismos que son expertos en ello por su oficio.

Un humilde marmitako marinero (patatas con bonito a tacos y chile, apio, puerro, pimiento $y$ tomate), comido en el País Vasco, puede ser una extraordinaria experiencia, como lo fue para mí en Cuzco la primera vez que degusté la t'inpusqa to "hervido"), el ruqutu ("pimiento") relleno o el chiri uchu ${ }^{16}$, nombres de muy profundas $y$ antiguas reminiscencias quechuas. Por lo demás, lo más auténtico es la preparación de los alimentos con

12 Es igualmente el engano, la picaresca, vla por la que se decantan aquellos que se valen de subterfugios para hacer una cocina tramposa y, generalmente, subida de precio.

$13 \mathrm{El}$ pensamiento de que la cocina incaica era perfecta, como nacida de un ideal científico y técnico, es solo producto de mentes calenturientas sobre el pasado utópico (caso de Antúnez de Mayulo 1981) o de investigadores, torpes e incapaces (caso de Hurtado Fuentes 2000), que lo son solo en apariencia.

14 Se trata de un procedimiento habitual. Por ejemplo en González Holguin la kalapurca o parirocro es "guisado, cozido con piedras encendidas". La primera de ellas es la llamada hoy día carapulca (vulgarmente carapulcra): "papa (cocida, sin descascarar, contada en láminas, de color marrón\})", que ha evolucionado a formas como "guisado \{de carnes, papa deshidratada, man y aji\})". Pari luqru ("locro caldeado") es un locro preparado por este procedimiento. He aqui las variedades más importantes que se registran de él: "(plato \{de carne, choclo, zapallo, patatas y aji\}), ruqru (luqlu); (guiso \{seco de zapallo, papa y queso\}), $k$ "irku; *fig.: (ajiaco), uchu".

15 Hay un plato universal, magistral pese a su sencillez, el pisto manchego, que contiene únicamente dos ingredientes: el tomate natural y los pimientos verdes, fritos, en aceite de oliva; sobre él, una dosis de huevo cocido granulado, calabacín picado y también cocido o lomo frito jugoso constituyen sendas variantes en las que todos creen estar ante la auténtica recéa. Lo mismo podrla decirse de la genial tortilla de patata (papa frita con aceite de oliva, en lárninas muy finas, y huevo batido que se cuaja con la patata en la sartén, penetrando en sus intersticios y recubriendo, sobre todo, sus dos caras), la cual admite, además, todo tipo de verduras fritas e incluso atún y que en ocasiones se condimenta con romero.

16 "Ajl frio", en quechua, plato fastuoso que se prepara para el Corpus Christi y cuyos ingredientes más sobresalientes son el cuy, las algas qucha yuyu, las huevas de pescado kaw-kaw y el rocoto. 
aquellos ingredientes que da, básicamente, la tierra: así es como aquí, al igual que en España, se ha logrado una gran riqueza culinaria regional, aunque Perú disfruta de una cierta ventaja: ha gozado hasta ahora de un mestizaje culinario (con España anteriormente y actualmente con China y Japón) que ha convertido a su cocina en una de las más variadas y espectaculares del mundo.

Acabadas estas reflexiones sobre cocina paleo-, meso- y neo-peruana, conviene resumir en un gráfico aproximativo los pilares en que se apoya, su hacer (ruway):

\section{REFERENCIAS BIBLIOGRÁFICAS}

Adelaar, Willem F.H. (1977): Tarma Quechua: grammar, texts, dictionary. Lisse, The Peter de Ridder Press.

Antúnez de Mayolo R., Santiago (1981): La nutrición en el Antiguo Perú. Lima, Banco Central de Reserva del Perú.

Bertonio, Ludovico (1612): Vocabvlario de la lengva aymara. Juli-Chucuito, Francisco del Canto.

Calvo Pérez, Julio (en prep.): Nuevo diccionario español-quechua / quechua-español (de próxima publicación por la Universidad de San Martín de Porres de Lima).

Espinoza Soriano, Waldemar (1987): Los Incas: economía, sociedad y Estado en la era del Tawuantinsuyu. Lima, Ediciones Amaru.

González Holguín, Diego (1608): Vocabvlario de la lengva general de todo el Perú, llamada lengua Qquichua, o del Inca. Lima, Francisco del Canto.

Hurtado Fuertes, Ciro (2000): La alimentación en el Tahuantinsuyo. Lima, Editorial San Marcos.

Inca Garcilaso de la Vega (1609-1617): Comentarios reales de los incas. Lisboa, Imprenta de Pedro Crasbeeck.
Lira, Jorge A. (1944): Diccionario kkechuwaespañol. Tucumán, Universidad Nacional de Tucumán.

Malpica Santisteban, Carlos (1965): Crónica del hambre en Perú. Lima, Francisco Moncloa Editores.

Middendorf, Ernst W. (1892): Wörterbuch des Runa Simi oder der Keshua-Sprache, en: Die einheimischen Sprachen Perus, vol. 2. Leipzig, F. A. Brockhaus.

Murra, John (1975): Formaciones económicas y políticas del mundo andino. Lima, Instituto de Estudios Peruanos.

Olivas Weston, Rosario (1998): La cocina en el virreinato del Perú. Lima, Escuela Profesional de Turismo y Hotelería, Universidad de San Martín de Porres.

Ortega Pérez, Fernando (1980): "La dicotomía caliente-frío en la medicina andina". En Debates de Antropología 5. Lima, Pontificia Universidad Católica del Perú.

Quesada C., Félix (1976): Diccionario quechua Cajamarca-Cañaris. Lima, Ministerio de Educación-Instituto de Estudios Peruanos.

Santo Thomás, Domingo de (1560): Lexicon, o vocabulario de la lengua general del Perv. VaIladolid, Francisco Fernández de Cordoua.

Taylor, Gérald (1974-1976): "Camay, camac y camasca dans le manuscript quechua de Huarochiri". En Journal de la Société des Américanistes, LXIII: 231-239.

Trier, Jost (1931): Der deutsche Wortschatz im Sinnbezirk des Verstandes. Die Geschichte eines sprachlichen Feldes. Heidelberg, Carl Winter.

Tschudi, Johann Jakob von (1853): Die Kechua Sprache, Sprachproben, Wörterbuch, 3 vol. Viena, Aus der Kaiserlich-Königlichen Hofund Staatsdruckerei. 
Valcárcel, Luis E. (1949): Historia de la cultura antigua del Perú. Lima, Imprenta del Ministerio de Educación.

Weber, David J. et al. (1998): Rimaykuna. Quechua de Huánuco. Lima, Instituto Lingüístico de Verano.
Yaranga Valderrama, Abdón (2003): Diccionario quechua español, runa simi-español. Lima, Université de Paris VIII - Biblioteca Nacional del Perú.

Zubritzki, Yuri A. (1975): Los incas-quechuas. Las etapas principales de la historia de un pueblo. Moscú, Ed. Progreso 